Supplement of Biogeosciences, 15, 6621-6635, 2018

https://doi.org/10.5194/bg-15-6621-2018-supplement

(C) Author(s) 2018. This work is distributed under

the Creative Commons Attribution 4.0 License.

(c) (1)

Supplement of

\title{
Impacts of temperature and soil characteristics on methane production and oxidation in Arctic tundra
}

\section{Jianqiu Zheng et al.}

Correspondence to: David E. Graham (grahamde@ornl.gov)

The copyright of individual parts of the supplement might differ from the CC BY 4.0 License. 
Table S1. Physicochemical characteristics of soils from FCP and HCP

\begin{tabular}{lllccccc}
\hline & Depth (cm) & Horizon & Water content & $\mathbf{p H ~ ( K C l )}$ & $\mathbf{F e}(\mathbf{I I})$ & Total C & C:N \\
& & & $(\mathbf{g ~ g - 1 )}$ & & umol g soil & $(\%)$ & \\
\hline FCP & $10-30$ & Organic & 0.44 & 4.24 & 9.72 & 18.56 & 18 \\
& $40-50$ & Transition & 2.48 & 4.86 & 50.20 & 5.80 & 16 \\
& $50-70$ & Permafrost & 3.95 & 4.95 & 68.95 & 30.83 & 18 \\
$\mathbf{H C P}$ & $10-30$ & Organic & 0.67 & 4.70 & 14.36 & 20.49 & 21 \\
& $50-70$ & Permafrost & 4.43 & 5.72 & 79.37 & 17.10 & 21 \\
\hline
\end{tabular}


Table S2. Fitted parameters for $\mathrm{CH}_{4}$ production with linear model $(C=A \times t+B)$.

\begin{tabular}{llllll}
\hline Horizon & Temperature & model & $\mathbf{A}$ & $\mathbf{B}$ & $\mathbf{R}^{\mathbf{2}}$ \\
\hline FCP Transition & +8 & Linear & 0.0685 & 0.0372 & 0.94 \\
& +4 & Linear & 0.0427 & 0.2133 & 0.81 \\
& -2 & Linear & 0.0166 & 0.1751 & 0.92 \\
FCP Permafrost & +8 & Linear & 0.0074 & 0.1039 & 0.79 \\
& +4 & Linear & 0.0060 & 0.0758 & 0.76 \\
& -2 & Linear & 0.0043 & 0.0895 & 0.73 \\
\hline
\end{tabular}


Table S3. Fitted parameters for $\mathrm{CO}_{2}$ production with Hyperbolic $(C=A \times t /(B+t))$, Linear $(C=A \times t+B)$, and Sigmoidal $\left(C=A \times t^{d} /\left(B^{d}+t^{d}\right)\right)$ models.

\begin{tabular}{lllllll}
\hline Horizon & Temperature & model & $\mathbf{A}$ & $\mathbf{B}$ & $\mathbf{d}$ & $\mathbf{R}^{2}$ \\
\hline FCP Organic & +8 & Hyperbolic & 545.19 & 53.60 & - & 0.85 \\
& +4 & Hyperbolic & 1588.17 & 545.18 & - & 0.91 \\
& -2 & Linear & 1.70 & 6.96 & - & 0.95 \\
FCP Transitional & +8 & Hyperbolic & 32.18 & 13.30 & - & 0.82 \\
& +4 & Hyperbolic & 28.35 & 16.11 & - & 0.78 \\
& -2 & Hyperbolic & 12.09 & 2.82 & - & 0.47 \\
FCP Permafrost & +8 & Hyperbolic & 48.52 & 14.63 & - & 0.84 \\
& +4 & Hyperbolic & 67.51 & 38.05 & - & 0.67 \\
HCP Organic & -2 & Hyperbolic & 45.80 & 32.55 & - & 0.87 \\
& +8 & Sigmoidal & 31.12 & 57.19 & 1.85 & 0.96 \\
HCP Permafrost & +4 & Sigmoidal & 17.10 & 44.42 & 2.32 & 0.87 \\
& -2 & Sigmoidal & 6.48 & 33.48 & 5.12 & 0.91 \\
& +4 & Sigmoidal & 3.69 & 50.56 & 25.43 & 0.90 \\
\hline
\end{tabular}


Table S4. Temperature sensitivities of soil anaerobic respiration and methanogenesis from FCP transition zone and permafrost

\begin{tabular}{|c|c|c|c|c|c|c|c|}
\hline \multirow[b]{2}{*}{ Horizon } & \multirow[b]{2}{*}{$\mathrm{T}\left({ }^{\circ} \mathrm{C}\right)$} & \multicolumn{4}{|c|}{$\mathrm{CO}_{2}$} & \multicolumn{2}{|l|}{$\mathrm{CH}_{4}$} \\
\hline & & $\begin{array}{c}\text { Rate }^{\mathrm{a}}\left(\mu \mathrm{mol} \mathrm{g}{ }^{-1} \text { day }^{-1}\right) \\
\text { Linear fitting }\end{array}$ & $\mathrm{Q}_{10}$ & $\begin{array}{c}\text { Rate }^{\mathrm{b}}\left(\mu \mathrm{mol} \mathrm{g}{ }^{-1} \text { day }^{-1}\right) \\
\text { Hyperbolic fitting }\end{array}$ & $\mathrm{Q}_{10}$ & Rate $\left(\mu \mathrm{mol} \mathrm{g}{ }^{-1}\right.$ day $\left.^{-1}\right)$ & $\mathrm{Q}_{10}$ \\
\hline \multirow{3}{*}{$\begin{array}{c}\text { FCP } \\
\text { Transition }\end{array}$} & -2 & 0.49 & 1.3 & 1.82 & 1.2 & 0.0166 & 4.1 \\
\hline & 4 & 0.48 & & 1.66 & & 0.0427 & \\
\hline & 8 & 0.64 & & 2.25 & & 0.0685 & \\
\hline \multirow{3}{*}{$\begin{array}{c}\text { FCP } \\
\text { Permafrost }\end{array}$} & -2 & 0.40 & 3.3 & 1.37 & 2.3 & 0.0043 & 1.7 \\
\hline & 4 & 1.00 & & 1.73 & & 0.0060 & \\
\hline & 8 & 1.31 & & 3.10 & & 0.0074 & \\
\hline
\end{tabular}


Table S5. Concentrations of Organic Acids* from FCP Transitional and Permafrost Layers.

\begin{tabular}{|c|c|c|c|c|c|c|c|}
\hline & \multirow[b]{2}{*}{$\begin{array}{c}\text { Incubation } \\
\text { days }\end{array}$} & \multicolumn{3}{|c|}{ FCP Transitional } & \multicolumn{3}{|c|}{ FCP Permafrost } \\
\hline & & $-2^{\circ} \mathrm{C}$ & $4^{\circ} \mathrm{C}$ & $8^{\circ} \mathrm{C}$ & $-2^{\circ} \mathrm{C}$ & $4^{\circ} \mathrm{C}$ & $8^{\circ} \mathrm{C}$ \\
\hline \multirow{3}{*}{ Formate } & 0 & & 0.68 & & & 1.68 & \\
\hline & 20 & 0.77 & 0.72 & 0.86 & 1.56 & 1.43 & 1.96 \\
\hline & 90 & $0.48 \pm 0.06$ & $0.52 \pm 0.02$ & $0.44 \pm 0.07$ & $0.99 \pm 0.2$ & $1.04 \pm 0.24$ & $1.14 \pm 0.05$ \\
\hline \multirow{3}{*}{ Acetate } & 0 & & 1.28 & & & 10.97 & \\
\hline & 20 & 1.44 & 1.76 & 1.89 & 10.95 & 9.78 & 12.94 \\
\hline & 90 & $2.00 \pm 0.44$ & $3.10 \pm 0.02$ & $3.27 \pm 0.07$ & $12.79 \pm 1.18$ & $15.29 \pm 0.75$ & $16.78 \pm 2.92$ \\
\hline \multirow{3}{*}{ Propionate } & 0 & & 0.49 & & & 3.82 & \\
\hline & 20 & 0.53 & 0.65 & 0.62 & 3.73 & 2.97 & 3.82 \\
\hline & 90 & $0.51 \pm 0.12$ & $0.51 \pm 0.03$ & $0.39 \pm 0.06$ & $8.9 \pm 0.33$ & $8.8 \pm 0.18$ & $10.1 \pm 0.41$ \\
\hline \multirow{3}{*}{ Butyrate } & 0 & & 0.10 & & & 1.74 & \\
\hline & 20 & 0.12 & 0.16 & 0.20 & 1.87 & 1.64 & 2.13 \\
\hline & 90 & $0.08 \pm 0.02$ & $0.15 \pm 0.01$ & $0.11 \pm 0.03$ & $1.92 \pm 0.11$ & $2.11 \pm 0.07$ & $2.17 \pm 0.13$ \\
\hline \multirow{3}{*}{ Oxalate } & 0 & & 0.09 & & & 0.23 & \\
\hline & 20 & 0.12 & 0.14 & 0.16 & 0.28 & 0.31 & 0.34 \\
\hline & 90 & $0.10 \pm 0.02$ & $0.11 \pm 0.00$ & $0.08 \pm 0.01$ & $0.23 \pm 0.03$ & $0.24 \pm 0.03$ & $0.26 \pm 0.01$ \\
\hline
\end{tabular}

* Results are presented in $\mu \mathrm{mol} \mathrm{g}{ }^{-1}$ (on per gram soil dry weight basis). The average and standard deviation are shown for triplicate soil samples incubated for 90 days. 
Figure S1. Geochemical properties of divided soil layers from (a) FCP and (b) HCP. Measured $\mathrm{Fe}(\mathrm{II})$ concentrations and soil porewater dissolved $\mathrm{CH}_{4}$ concentrations are plotted and data points corresponding to soil segments used for incubations are circled in red.
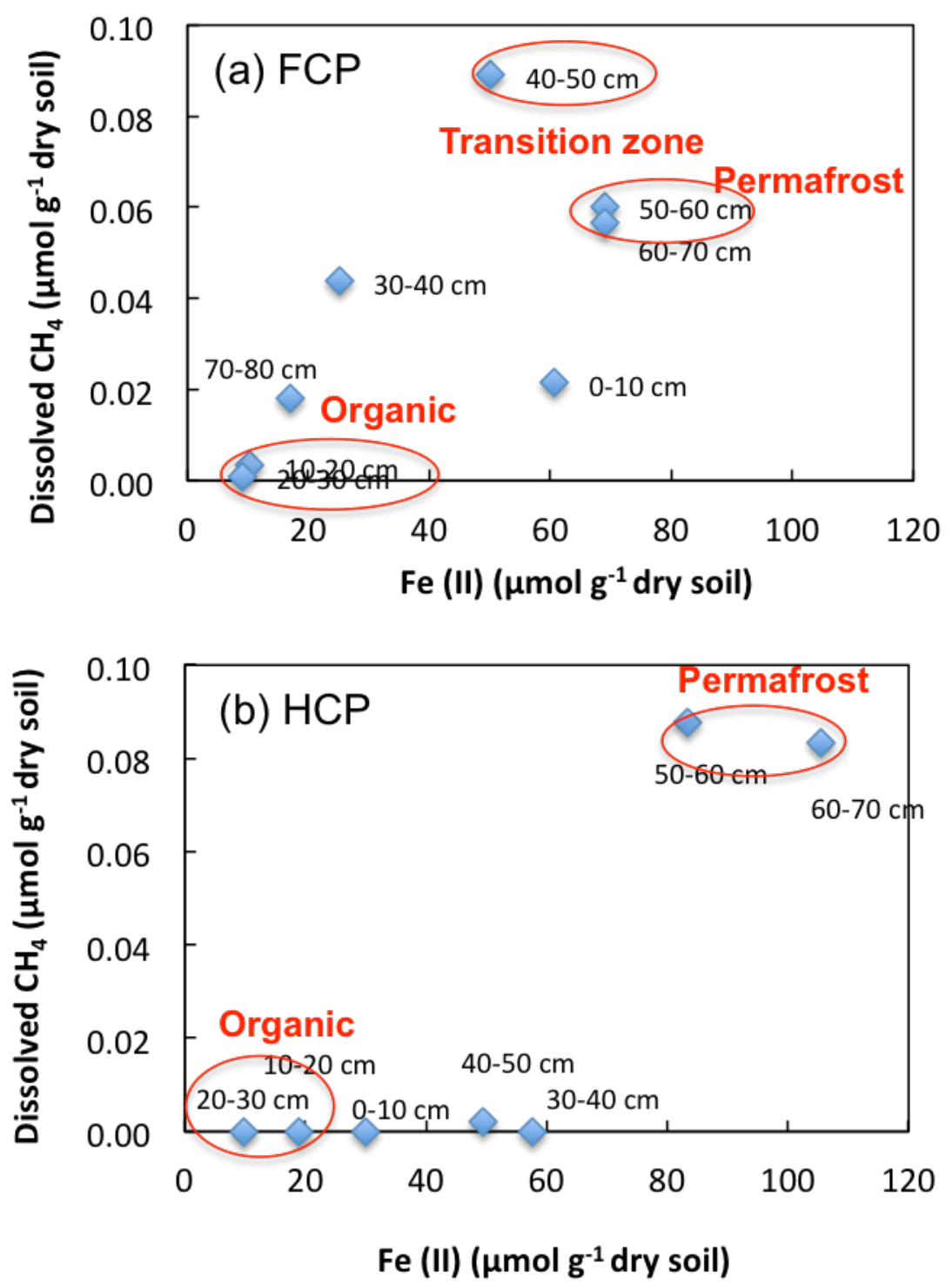
Figure $\mathrm{S} 2 . \mathrm{CH}_{4}$ production in soil microcosm from active and permafrost horizons of HCP, and active horizon of FCP.

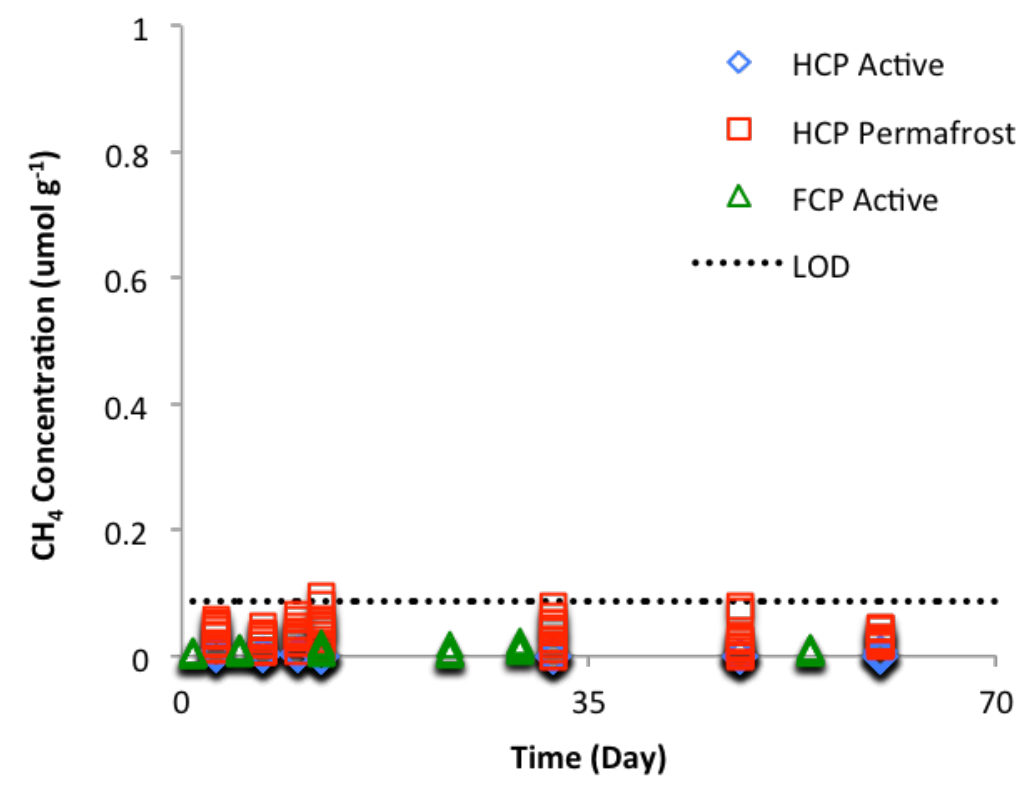

